\title{
Philosophiques
}

\section{Discours prononcé à l'occasion de la remise du Prix André Laurendeau 1992}

\section{François Duchesneau}

Volume 21, numéro 1, printemps 1994

URI : https://id.erudit.org/iderudit/027256ar

DOI : https://doi.org/10.7202/027256ar

Aller au sommaire du numéro

Éditeur(s)

Société de philosophie du Québec

ISSN

0316-2923 (imprimé)

1492-1391 (numérique)

Découvrir la revue

Citer ce document

Duchesneau, F. (1994). Discours prononcé à l'occasion de la remise du Prix André Laurendeau 1992. Philosophiques, 21(1), 191-195.

https://doi.org/10.7202/027256ar d'utilisation que vous pouvez consulter en ligne.

https://apropos.erudit.org/fr/usagers/politique-dutilisation/ 


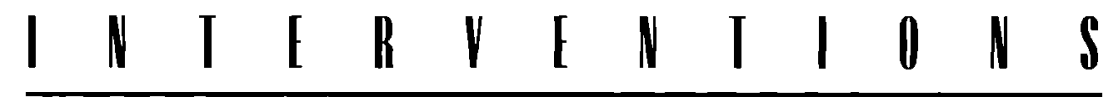

\section{Discours prononcé à l'occasion de la remise du PRIX ANDRÉ LAURENDEAU 1992}

\section{par \\ François Duchesneau}

Je tiens à témoigner ma gratitude à ACFAS qui me décerne aujourd'hui le Prix André Laurendeau. Je suis très sensible à l'honneur qui mest fait.

Une occasion comme celle-ci incite à une forme d'introspection qu'on ne pratique que très rarement à l'égard du profil intérieur de sa propre carrière. Certes, le jury s'est essentiellement intéressé au profil externe, à l'image publique du chercheur. Appartiennent à cette image les travaux savants, et en premier lieu les publications et les réalisations de recherche, dans une certaine mesure aussi le rôle institutionnel que le lauréat a pu jouer dans son milieu et les fonctions qu'il a assumées. En un mot, le prix sanctionne une contribution significative à l'avancement des connaissances.

La statue intérieure, pour employer la merveilleuse métaphore de François Jacob, est plus difficile à décrire. Il faut un sculpteur particulièrement habile et expérimenté pour la tirer de la gangue qui l'enveloppe et la dissimule aux regards. D'ailleurs elle n'est jamais préformée, elle se forme peu à peu et se reforme constamment sous l'influence d'une multitude de déterminations: telle la statue de Condillac conjugué au moule intérieur de Buffon - n'est-ce point là l'une des sources possible de cette métaphore ? - notre statue intérieure surgit de ses dispositions natives mais elle se développe en tirant parti des circonstances et sous l'influence des forces qu'exerce le milieu ambiant. Le processus est une Ausbildung, une épigenèse, qui fait appel à des 
dispositions et à des pouvoirs architectoniques inhérents, mais qui opère dans un contexte de déterminations externes.

Je ne jouerai pas aujourd'hui les architectes introspectifs; je me contenterai plutôt d'indiquer quelques élements contextuels sans lesquels le profil intérieur de ma recherche et de mon action académique manquerait de raison suffisante, et ne pourrait donc constituer une vérité de fait. Privé de ma propre vérité contingente comme chercheur, la notion de moi-même risquerait de n'être qu'une simple possibilité abstraite, un ens rationis dangereusement évanescent. Mais la tâche de prouver une vérité de fait est ardue: comme Leibniz l'indique, une vérité de fait supposerait une résolution analytique infinie de ses raisons internes. La solution pragmatique est de partir des faits et de tenter d'expliquer les phénomènes à l'aide d'une hypothèse raisonnable. Dans cette perspective, je me contenterai de vous offrir quelques éléments de représentation concrète que l'on ne saurait sans doute percevoir à la lecture de mon curriculum vitæ.

La Sorbonne que j'ai connue comme étudiant de premier et de deuxième cycle, était hautement mandarinale. C'était avant 68. Même si j'y ai beaucoup appris, les courants méthodologiques de la philosophie régnante, phénoménologique et herméneutique, ne m'ont guère marqué. Par contre, j'ai pris le plus vif intèrêtà la psychologie, à la sociologie et à la logique. J'ai touché à l'éthique, qui m'a alors rebuté par son manque de rigueur - je suis depuis revenu de ce préjugé, et j'ai même soutenu le projet d'une vaste enquête sur l'éthique appliquée lorsque j'étais président de la Fédération canadienne des études humaines. Jai enfin découvert l'épistémologie en préparant de façon très personnelle le concours de l'agrégation. Comme l'empirisme était alors fort décrié dans le milieu philosophique français - il l'est toujours d'ailleurs mon intérêt s'est tourné, peut-être par esprit de paradoxe, vers la théorie empiriste de la connaissance considérée en ses fondements historiques. En négociant un sujet de thèse de doctorat avec Yvon Belaval, j’ai rencontré unè idée séminale : la théorie empiriste de la connaissance, qui est une veritable théorie de la connaissance contrairement à ce qu'on m'avait appris, décentre le discours philosophique en l'écartant de la métaphysique. Suivant cette « révolution copernicienne » avant celle de Kant, l'analyse philosophique sert désormais d'instrument pour ajuster et justifier les nouvelles méthodologies scientifiques que signifie le concept d'experimental philosophy. Or une part considérable des problématiques actuelles de la discipline philosophi-que ne s'explique que par ce glissement stratégique de fonction. Et c'est là, à mon avis, un objet d'étude sur lequel on s'est trop peu penché. 
Par mutations successives, une telle idée-force a motivé la plupart de mes travaux jusqu'à ce jour. Mes livres marquent les moments de synthèse provisoire issus de ces mutations : L'empirisme de Locke en 1973. La physiologie des Lumières. Empirisme, modèles et théories en 1982, Genèse de la théorie cellulaire en 1987, sans compter les deux ouvrages actuellement sous presse : Leibniz et la dynamique et Leibniz et la méthode de la science. L'ensemble de recherches dont ces publications sont le produit et l'expression la plus manifeste, se concentre autour de sujets à la frontière de l'histoire et de la philosophie des sciences. Il s'agit de découvrir quels principes épistémologiques plus ou moins explicites ont présidè à la formation des théories de la science et comment réciproquement les methodologies de la science imposent des contraintes et des objets à l'analyse philosophique. Certes, à l'arrière-plan de ces recherches, prévaut un choix stratégique relatif à la carrière philosophique.

Comme vous le savez, Descartes avait choisi comme devise : Larvatus prodeo, que l'on pourrait traduire J'avance masqué. Cette devise convenait à un chercheur solitaire oeuvrant en marge de toute institution, professant la plus grande réticence à dévoiler sa pensée aux intelligences imparfaites de ses contemporains, par ailleurs extrêmement jaloux de sa gloire auprès des générations futures. Cette figure mythique du philosophe se situant hors de la Cité fut érigée en paradigme à l'époque romantique; et ce paradigme règne encore sur notre représentation idéologique du philosophe. Puisque j'ai décidé de vous parler de certaines déterminations concrètes qui ont influé sur mon profil de recherche, je ne saurais omettre de justifier quelque peu mon implication dans l'administration académique et universitaire. Javoue que je me sens à cet égard plus en harmonie avec d'autres modèles qu'avec le modèle cartésien. La polyvalence de Locke et de Leibniz, sur lesquels j'ai beaucoup travaillé, m'a toujours particulièrement séduit. Le premier, médecin, homme politique, ideologue de la Révolution anglaise, a détenu nombre de postes, dont le très important Secrétariat pour le commerce et les colonies. Le second, polymathe par excellence, fondateur d'académies et de revues savantes, philosophe, théologien, juriste, mathématicien, physicien, historien, philologue, s'est illustre dans la carrière diplomatique et dans divers domaines de gestion gouvernementale dont il a percu l'importance, entre autres la politique scientifique, champ disciplinaire que nous croyons à tort avoir inventé dans le passé le plus récent. Évidemment, ce qui se dessine alors c'est le profil institutionnel du philosophe des Lumières qui tire de la science, œuvre collective et non plus individuelle, les objectifs et les moyens d'une action qui dépasse elle aussi la sphère de l'individualité stricte. Nous sommes les héritiers de ce modèle du philosophe comme de l'autre. 
Pour ma part, je confesse volontiers ma très forte préférence pour le modèle de l'Aufklärer. Aussi ne serez-vous point étonnés d'apprendre que mes fonctions me sont toujours apparues comme des défis intellectuels, sans doute parfois un peu lourds, mais susceptibles de mettre mon empirisme à l'épreuve des faits. Je perçois surtout très difficilement que la recherche universitaire, surtout philosophique, puisse se développer comme en vase clos, à l'abri de toute implication institutionnelle, voire en marge de tout enseignement : ce serait alors de la spéculation purement gratuite; et comme telle, la philosophie spéculative manque à mon sens de raison suffisante : elle mapparaît comme un discours stérile. Certes, certains esprits plus enclins à la métaphysique, pourraient m'objecter que la pensée philosophique est plus profonde et plus radicale que le principe même de raison suffisante, ou encore, qu'elle doit surplomber d'une vertigineuse hauteur toute expérience possible. Comme vous l'aurez compris, je ne suis guère sympathique à cette vision éthérée et selon moi improductive de la philosophie.

Certes, il est un prix à payer si l'on adhère à cet éthos de la philosophie: il en résulte que la carrière ne peut se concevoir suivant un seul axe, celui de la recherche spécialisée. La conciliation d'une pluralité de cheminements, à la fois d'ordre administratif et d'ordre académique, doit s'opérer. Or l'expérience m'a appris que cette conciliation s'opère de façon beaucoup plus imprévisible qu'une reconstitution adroite ne pourrait le laisser croire après coup. Il s'agit donc d'un véritable développement: le profil intérieur d'une carrière de recherche de ce type passe de mutation en mutation sans que l'on soit en mesure d'en décrire autres choses que des traits relativement disparates qui semblent néanmoins vouloir se concilier et se combiner en une synthèse à la limite dans un horizon futur.

Je terminerai donc sur une idée leibnizienne centrale aux projets d'encyclopédie et merveilleusement conforme au thème du présent congrès. Voici cette idée : le développement externe et collectif des connaissances peut s'interpréter comme le reflet multiple et intégré des cheminements internes et individuels les plus divers, par lesquels se réalise la science. Et je citerai un fragment de projet d'encyclopédie dont je me plais à penser que Leibniz le destinait à quelque ministre du RoiSoleil, ce qui ne serait pas sans humour :

Le genre humain considéré par rapport aux sciences qui servent à notre bonheur, me parait semblable à une troupe de gens, qui marchent en confusion dans les ténebres, sans avoir ni chef, ni ordre, ni mot, ni autres marques pour régler la marche et pour se reconnaître [... Il n'appartient à aucun mortel d'allumer un flambeau capable de chasser cette obscurité; les sectes et les chefs de secte ne servent quä nous séduire comme les fausses lueurs des feux follets, et 
il est réservé au soleil de nos âmes de nous éclairer entièrement, mais dans une autre vie. Cependant ce que nous pouvons faire ici, c'est de marcher de concert et avec ordre, de partager les routes, de faire reconnaitre les chemins et de les raccommoder; enfin d'aller lentement, mais d'un pas sûr et ferme le long de ce ruisseau d'eau vive des connaissances simples et claires, qui prend sa source parmi nous [..] se grossit peu à peu, et fait augmenter nos connaissances, et qui nous mène enfin lauxl plus importantes verités de pratique [... (GP, vII, I57).

\section{Université de Montréal}

